\title{
Response of AGATA segmented HPGe detectors to gamma rays up to $15.1 \mathrm{MeV}$
}

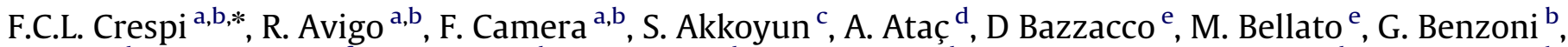

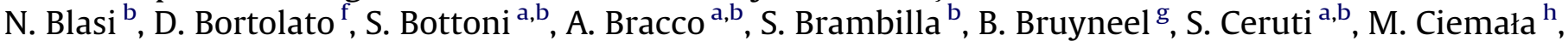 \\ S. Coelli ${ }^{b}$, J. Eberth ${ }^{\mathrm{i}}$, C. Fanin ${ }^{\mathrm{e}}$, E. Farnea ${ }^{\mathrm{e}}$, A. Gadea $^{\mathrm{j}}$, A Giaz $^{\mathrm{a}, \mathrm{b}}$, A. Gottardo ${ }^{\mathrm{k}, \mathrm{f}}$, H. Hess ${ }^{\mathrm{i}}$, M. Kmiecik ${ }^{\mathrm{h}}$, \\ S. Leoni ${ }^{\mathrm{a}, \mathrm{b}}$, A. Maj ${ }^{\text {h }}$, D. Mengoni ${ }^{\text {l,e }}$, C. Michelagnoli ${ }^{\mathrm{k}, \mathrm{e}}$, B. Million ${ }^{\mathrm{b}}$, D. Montanari ${ }^{\mathrm{e}}$, R. Nicolini ${ }^{\mathrm{a}, \mathrm{b}}$, \\ L. Pellegri ${ }^{\mathrm{a}, \mathrm{b}}$, F. Recchia ${ }^{\mathrm{k}, \mathrm{e}}$, P. Reiter ${ }^{\mathrm{i}}$, S. Riboldi ${ }^{\mathrm{a}, \mathrm{b}}$, C.A. Ur ${ }^{\mathrm{e}}$, V. Vandone $^{\mathrm{a}, \mathrm{b}}$, J.J. Valiente-Dobon ${ }^{\mathrm{f}}$, \\ O. Wieland ${ }^{\text {b }}$, A. Wiens ${ }^{\mathrm{i}}$, The AGATA Collaboration
}

\footnotetext{
a Dipartimento di Fisica, Università di Milano, Milano I-20133, Italy

${ }^{\mathrm{b}}$ INFN, Sezione di Milano, Milano I-20133, Italy

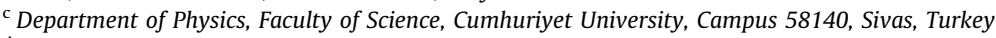

d Department of Physics, Faculty of Science, Ankara University, Tandoðan 06100, Ankara, Turkey

e INFN, Sezione di Padova, Padova I-35131, Italy

${ }^{\mathrm{f}}$ INFN, Laboratori Nazionali di Legnaro, Legnaro IT-35020, Italy

${ }^{g}$ CEA-Saclay DSM/IRFU/SPhN, Gif-sur-Yvette 91191, France

${ }^{\mathrm{h}}$ The Niewodniczanski Institute of Nuclear Physics, Polish Academy of Sciences, 31-342 Krakow, Poland

${ }^{\mathrm{i}}$ Institut für Kernphysik, Universität zu Köln, Zülpicher Str. 77, Köln D-50937, Germany

j IFIC, CSIC-University of Valencia, Valencia ES-46071, Spain

${ }^{\mathrm{k}}$ Dipartimento di Fisica e Astronomia dell'Universitàdi Padova, Padova I35131, Italy

${ }^{1}$ School of Engineering, University of the West of Scotland, Paisley PA1 2BE, United Kingdom
}

\section{A R T I C L E I N F O}

\section{Article history:}

Received 21 September 2012

Received in revised form

11 December 2012

Accepted 13 December 2012

Available online 20 December 2012

\section{Keywords:}

AGATA

Gamma-ray spectroscopy

Gamma-ray tracking

HPGe detectors

Pulse-shape and gamma-ray tracking

algorithms

Semiconductor detector performance and simulations

\begin{abstract}
A B S T R A C T
The response of AGATA segmented HPGe detectors to gamma rays in the energy range 2-15 MeV was measured. The $15.1 \mathrm{MeV}$ gamma rays were produced using the reaction $\mathrm{d}\left({ }^{11} \mathrm{~B}, \mathrm{n} \gamma\right)^{12} \mathrm{C}$ at $E_{\text {beam }}=19.1 \mathrm{MeV}$, while gamma rays between 2 and $9 \mathrm{MeV}$ were produced using an Am-Be-Fe radioactive source. The energy resolution and linearity were studied and the energy-to-pulse-height conversion resulted to be linear within $0.05 \%$.Experimental interaction multiplicity distributions are discussed and compared with the results of Geant 4 simulations. It is shown that the application of gamma-ray tracking allows a suppression of background radiation caused by n-capture in Ge nuclei. Finally the Doppler correction for the $15.1 \mathrm{MeV}$ gamma line, performed using the position information extracted with Pulse-shape analysis is discussed.
\end{abstract}

(c) 2012 Elsevier B.V. All rights reserved.

\section{Introduction}

In many in-beam gamma spectroscopy experiments the detection of high-energy gamma rays in the $10-20 \mathrm{MeV}$ range is of primary importance (see e.g. Refs. [1-5]). The limited size of the presently available HPGe crystals (up to $\sim 400 \mathrm{~cm}^{3}$ ) affects the

\footnotetext{
* Corresponding author at: Università di Milano, Dipartimento di Fisica, INFN, Sezione di Milano, via celoria 16, Milano I-20133, Italy. Tel.: + 390250317261.

E-mail address: fabio.crespi@mi.infn.it (F.C.L. Crespi).
}

possibility to detect the full energy deposition of such highenergy photons. However, large detection volumes (and, consequently, large detection efficiencies) can be obtained by using composite germanium detectors, namely using multiple crystals within the same cryostat, as was done in the past with the Clover detectors [6] and with the EUROBALL Cluster detectors [7-10]. The response function of such composite detectors was investigated up to $15 \mathrm{MeV}$ [11-13]. The additional benefit of generating large detection volumes packing several small crystals together is the reduction of the Doppler broadening of photons emitted in-flight, owing to the finite solid angle subtended by each crystal in case 
the photons are emitted from recoiling nuclei. With the new generation high-resolution gamma-ray spectrometers like AGATA [14-16] and GRETA $[17,18]$, the HPGe crystals are operated in position-sensitive mode through a combination of electrical segmentation of the outer electrodes, digital electronics and sophisticated Pulse Shape Algorithms [19-28]. The energy and direction of the individual photons are extracted through dedicated gamma-ray tracking algorithms [29-32]. It should be remarked that the individual interaction points are extracted with sub-segment precision, which experimentally turns out to be better than a three dimensional (3D) Gaussian with $5 \mathrm{~mm}$ FWHM in each direction (see for instance Refs. [33-36]). In order to achieve this goal, remarkable effort has been devoted to the characterization of highly-segmented HPGe detectors [37-52], and the possibility to improve the performances of a gammaray spectrometer at high energies using accurate 3D position information was first proposed in Ref. [53].

The performance of the Advanced GAmma-ray Tracking Array (AGATA) detectors with in-beam tests were discussed in Ref. [33-36]. These studies, however, were limited to gamma-rays energies up to $4 \mathrm{MeV}$. The present work provides the first detailed study of the response of AGATA detectors to gamma-rays up to 15.1 MeV. This study represents an important test of the AGATA detectors for the measurement of high-energy gamma rays, in terms of energy resolution, tracking efficiency and performance of the PSA algorithms. This aspect will be important in the forthcoming experimental campaign with relativistic beams [54] at GSI, where, the energies of the gamma-rays emitted in flight can be significantly Doppler shifted toward higher values.

In Section 2 we describe the experimental set-up, the Am-Be-Fe source calibrations and the in-beam test, while in Section 3 the results concerning detector energy resolution and linearity as a function of the gamma-ray energy are presented. Experimentally extracted interaction multiplicity distributions are shown and compared with Geant4 [55-57] simulations in Section 4. Finally, in Section 5 we discuss the Doppler correction using the PSA and gamma-ray tracking for the $15.1 \mathrm{MeV}$ gamma line.

\section{In-beam test and Am-Be-Fe source measurement}

The reaction used to produce the $15.1 \mathrm{MeV}$ gamma-ray was $\mathrm{d}\left({ }^{11} \mathrm{~B}, \mathrm{n} \gamma\right){ }^{12} \mathrm{C}$ at $E_{\text {beam }}=19.1 \mathrm{MeV}$.

$A{ }^{11} \mathrm{~B}$ beam with an energy of $45 \mathrm{MeV}$ from the Legnaro XTU Tandem accelerator was degraded to $19.1 \mathrm{MeV}$ using an Au foil in front of the target $\left(29 \mathrm{mg} / \mathrm{cm}^{2}\right)$. The reaction populates the resonance state at $15.1 \mathrm{MeV}$ in ${ }^{12} \mathrm{C}$ nucleus which is produced with a $\mathrm{v} / \mathrm{c} \sim 5 \%$. This state decays directly to the ground state (with a branching ratio of $92 \%$ [58]) by emitting a single M1 gamma-ray with an energy of $15.1 \mathrm{MeV}$ [59-61]. The target was made of $C_{32} D_{66}$ (dotriacontane-d66) with a thickness of $490 \mu \mathrm{g} /$ $\mathrm{cm}^{2}$, deposited on a $0.1 \mathrm{~mm}$ thick tantalum backing. Both the recoiling nuclei and the beam were stopped in the target backing. The gamma rays produced in the reaction were measured with two AGATA triple clusters, which were placed at a distance of $13.5 \mathrm{~cm}$ from the target. The AGATA electronics was set in order to have $0-20 \mathrm{MeV}$ dynamic range, for each segment and for the cores. The trigger condition did not require any coincidence with other detectors. One large volume cylindrical 3.5 in. $\times 8$ in. $\mathrm{LaBr}_{3}$ :Ce detector, having larger efficiency as compared to one single AGATA crystal was added to the experimental set-up for monitoring purposes and operated using an independent acquisition system (upper panel of Fig. 1) [62,63].

The detectors were calibrated using an Am-Be-Fe source. The Am-Be-Fe source was placed into a $3 \times 3 \mathrm{~cm}$ hole drilled in an iron slab of $7 \times 7 \times 20 \mathrm{~cm}$ and surrounded by paraffin wax in a
$20 \times 20 \mathrm{~cm}$ cylindrical shape (see the bottom panel of Fig. 1 ). The neutrons from the Am-Be-Fe source were thermalized in the paraffin housing and then captured in iron producing gamma rays of energies up to $9.3 \mathrm{MeV}$.

The gamma-ray spectrum acquired using the Am-Be-Fe source is displayed in Fig. 2 and the gamma lines used for the analysis are labeled with the corresponding reaction. These data were used to calibrate and check the linearity and energy resolution of the AGATA detectors. The average counting rate per crystal was $0.9 \mathrm{kHz}$ during the source measurement, and $1.2 \mathrm{kHz}$ in the in-beam test.

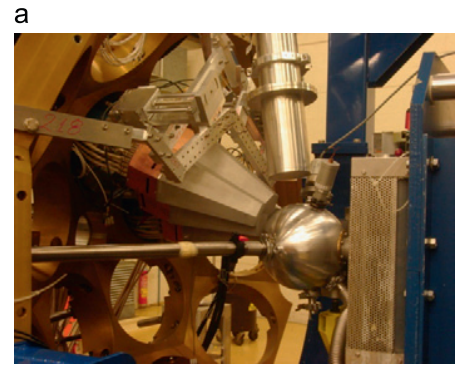

b

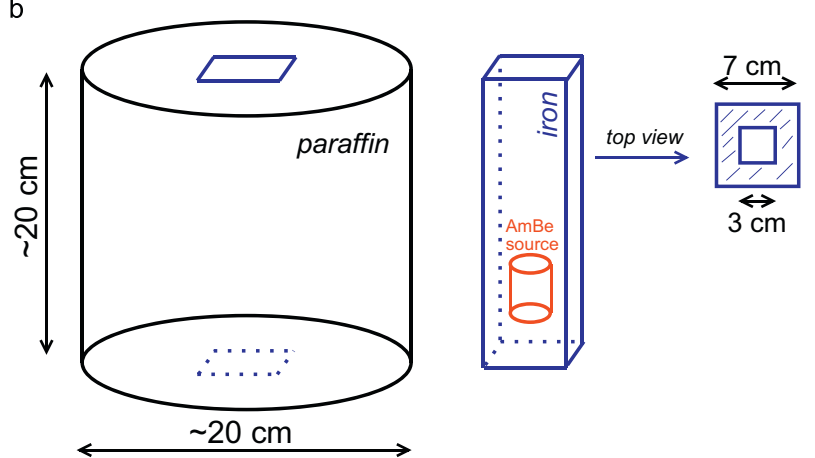

Fig. 1. Upper panel: The experimental set-up consisting of two AGATA triple clusters and one $3.5^{\prime \prime} \times 8$ " cylindrical $\mathrm{LaBr}_{3}$ :Ce scintillation detector. Lower panel: schematic representation of the Am-Be-Fe source.
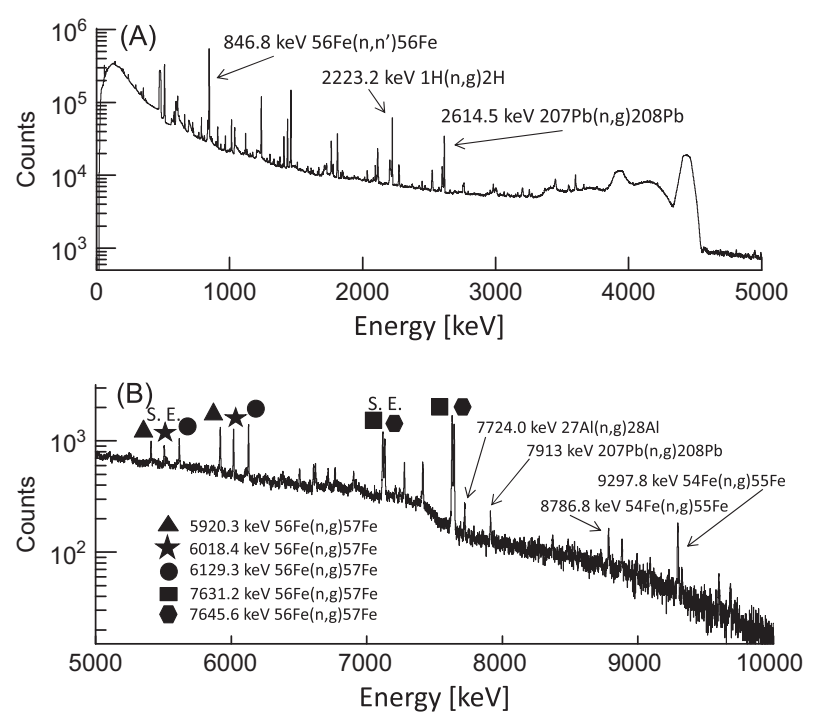

Fig. 2. Gamma-ray energy spectrum measured with the Am-Be-Fe source in the 0-5 MeV range (panel $\mathrm{A}$ ) and in the $5-10 \mathrm{MeV}$ range (panel $\mathrm{B}$ ). The gamma lines used for the analysis are labeled by the corresponding reaction. 


\section{Energy resolution and linearity}

In Fig. 3 the relative energy resolution (i.e. FWHM/E $E_{\text {gamma }}$ ) as a function of the gamma-ray energy is displayed. The data associated to the single crystal showing the best performance are reported with empty black circles. The black triangles represent, instead, the energy resolution obtained by summing the energies detected by the crystals that fired in each event (addback).

All the spectra analyzed in this section were extracted without using any kind of filter, but only summing the energy measured in each segment; this procedure is feasible because of the low gamma-ray multiplicity(see e.g. Section 4). These segment energies are extracted at pre-processing level by applying the moving window deconvolution (MWD) algorithm $[64,65]$ to the incoming data streams. In this way it was possible to perform (offline) a fine gain matching of all segments. This latter procedure turned out to be extremely important especially when high-energy gamma rays are involved. In addition, for each crystal, the sum energy of the segments was forced to be equal to the energy extracted from the core signal, in order to recover the segment energy resolution, degraded by neutron damage [66]. It is important to mention that a more sophisticated method to recover neutron damage in segmented HPGe detectors, exploiting position information provided by PSA algorithms, was recently developed [67]. However, such a procedure is not expected to provide a significant improvement for the specific case of high-energy gamma rays, considered in this work.

As can be seen from Fig. 3, the experimental data follow the expected $E^{-1 / 2}$ trend (indicated by the black dashed line). The FWHM of the highest-energy gamma line (i.e. $9297.8 \mathrm{keV}$ ) is $6.1 \mathrm{keV}$ in the case of the single crystal with the best performances, and $7.6 \mathrm{keV}$ for the add-back case. The energy resolution obtained for the $15.1 \mathrm{MeV}$ gamma emitted in the in-beam test is not displayed since the FWHM of the peak is, in this case, dominated by the Doppler broadening induced by the reaction mechanism (see Section 5 for details). However, considering the trend showed by the data displayed in Fig. 3, an intrinsic resolution of the order of $10 \mathrm{keV}$ is expected at the energy of $15 \mathrm{MeV}$.

In the following we present the study of the linearity for the energy to pulse-height conversion up to $15 \mathrm{MeV}$.

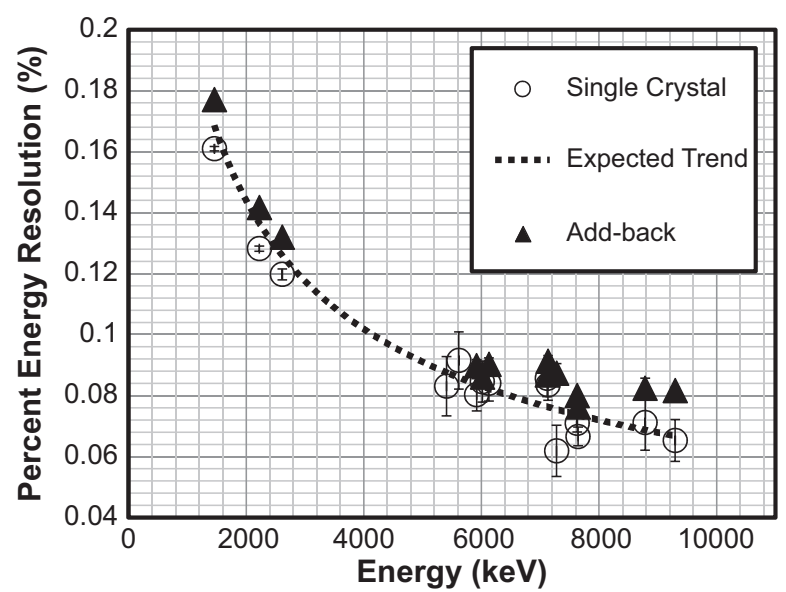

Fig. 3. Relative energy resolution of the AGATA detectors is given for the Am-Be-Fe source data. The data for the best performing single detector are shown by empty black circles. The black triangles represent instead the energy resolution for the add-back procedure, performed among all crystals that fired in each event. The experimental data follow the expected $E^{-1 / 2}$ trend (indicated by the dashed black line).

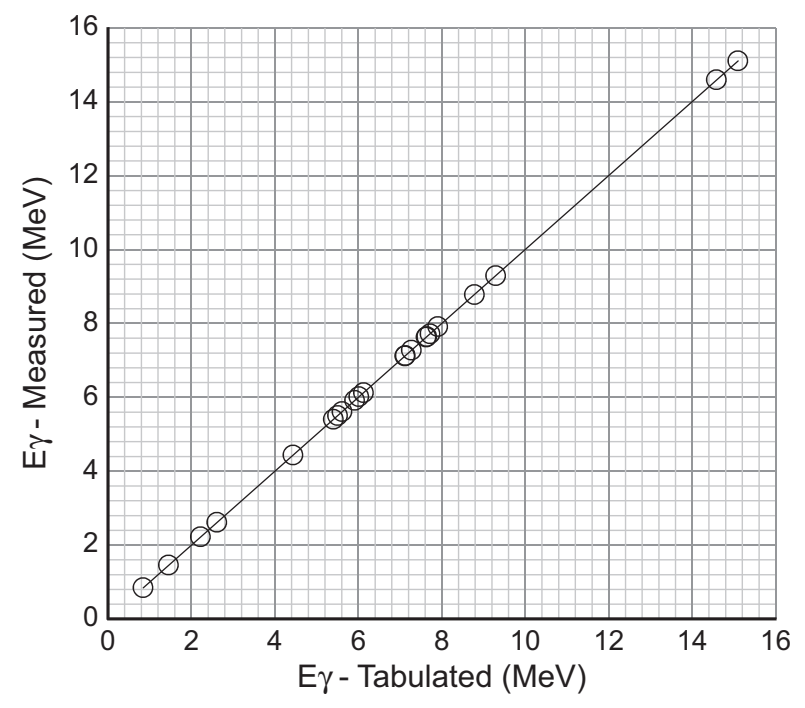

Fig. 4. Tabulated energy versus measured energy for gamma lines of the Am-Be-Fe source and for the 4.4 and $15.1 \mathrm{MeV}$ gammas from the in-beam test.

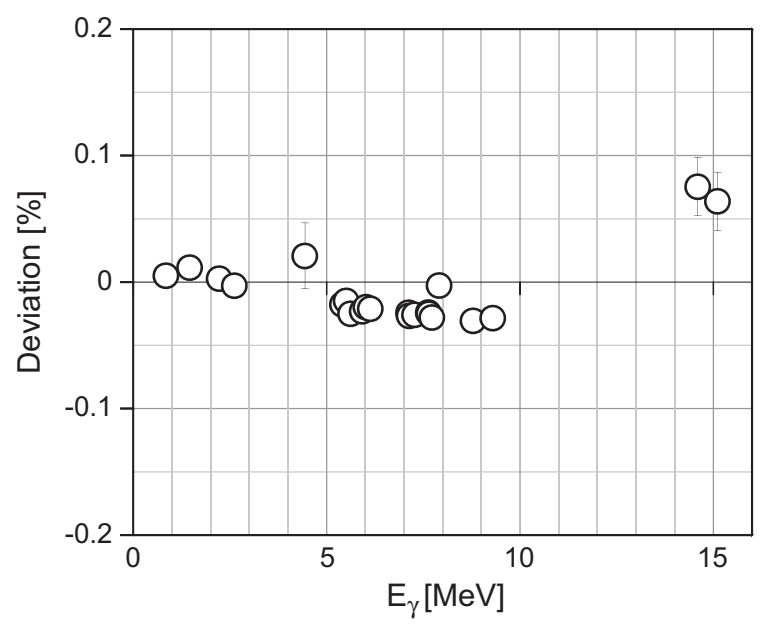

Fig. 5. Deviation of the measured energies from the tabulated energy for each gamma line of the Am-Be-Fe source and for the 4.4 and $15.1 \mathrm{MeV}$ gammas from the in-beam test. If not displayed, error bars are smaller than the symbol size.

The plot in Fig. 4 displays the measured energy versus the tabulated energy for gamma lines of the Am-Be-Fe source and for the 4.4 and $15.1 \mathrm{MeV}$ gamma rays from the in-beam reaction. The measured energy is obtained with a linear calibration using the 1173 and $1332 \mathrm{keV}$ lines of a ${ }^{60} \mathrm{Co}$ source. In addition, a fine gain matching of the detector segments was performed. This procedure allowed us to refine the calibration coefficients obtained using only ${ }^{60} \mathrm{Co}$ source. The gain matching coefficients for each single segment were extracted performing linear interpolation of the $846.8,2223.2$ and $2614.5 \mathrm{keV}$ gamma lines, on spectra incremented only if the highest energy release in the event was registered in the selected segment.

Percent deviation of the experimental data from tabulated energies is reported in Fig. 5 as a function of energy. The deviation is defined as the difference between measured and tabulated energy divided by measured energy (Deviation $=\left(E_{\text {meas }}-E_{\mathrm{tab}}\right)$ / $E_{\text {meas }}$ ). As expected data corresponding to gamma rays emitted in-flight show larger error bars. It is found that the total deviations from ideal linearity are lower than $0.1 \%$ in the energy range 2-15 MeV. Such results are consistent with those reported in [13] for the case of EUROBALL [7-10] clusters. 


\section{Multiplicity distributions}

In this section, the multiplicity distributions of AGATA clusters, crystals and segments are discussed. The results were extracted with data from the Am-Be-Fe source measurement described in Section 2. Full Energy Peak (F.E.P.) events are selected, for each gamma-ray energy, imposing a gate centered on the corresponding full energy absorption peak in the gamma spectrum; background events are selected instead imposing two gates respectively corresponding to the two intervals adjacent to the left and right boundary of the gate used for selecting F.E.P. events. Unless otherwise specified, the plots are produced without applying any filter to the data (e.g. gamma-ray tracking algorithm).

In Table 1 the cluster multiplicity ( $\left.M_{\text {clust }}\right)$ distributions for full energy peak (FEP) and background events are listed. The AGATA HPGe crystals are packed in common cryostats in groups of three, each one of these group is referred to as a (triple) cluster; we define $M_{\text {clust }}$ as the number of triple clusters (i.e. cryostats) sharing the energy release. In particular, when $M_{\text {clust }}=1$ it is intended that the energy release is confined to crystals of one single cluster. The table clearly shows a general increase, with gamma-ray energy, of the fraction of the events in which the energy release is shared between both clusters $\left(M_{\text {clust }}=2\right)$. In addition, background events show a larger percentage of $M_{\text {clust }}=2$ events as compared to full energy peak ones. The same behavior can be observed in Fig. 6, which displays the crystal multiplicity distributions for full energy peak (bottom panel) and background events (top panel). Such a behavior, in the case of the Am-Be-Fe source data, is due to the fact that background events originate mostly from neutron interactions in HPGe detectors and subsequent neutron induced gamma emission. These events are expected to have in average a larger multiplicity as compared to gamma-ray FEP events, leading to the same total energy release in the HPGe detectors. This can be attributed to the presence of additional interaction points associated to inelastic neutron scattering with Ge nuclei [68] and to the multiplicity of gamma rays emitted following the de-excitation of Ge nuclei.

Fig. 7 displays the centroid of the segment multiplicity distributions, as a function of gamma-ray energy, for FEP (top panel) and background (bottom panel) events. In addition, the segment multiplicity distributions extracted using a simple add-back algorithm (i.e. summing up the energies of all the interactions in the two clusters) are compared with those extracted applying the gamma-ray tracking alogirthm [69]. It should be mentioned here that AGATAdetectors can provide also sub-segment information concerning interaction number distributions (see e.g. Ref. [25]). Nevertheless, in this specific study the used algorithm [19] provides a single interaction point in the segment where a net charge deposition took place, implying that the multiplicity distributions of interaction points and of segments necessarily coincide.

By looking at Fig. 7 one notices that even though the general behavior is identical up to $7 \mathrm{MeV}$, for higher energies a clear deviation between the two curves appears. This effect can be attributed to the rejectionof neutron captureevents performed by the tracking algorithm [69]. In fact this kind of events are characterized by high multiplicity of gamma rays emitted following Ge nuclei de-excitation. An example of this background rejection is shown in Fig. 8 where the line at $10.196 \mathrm{MeV}$ is
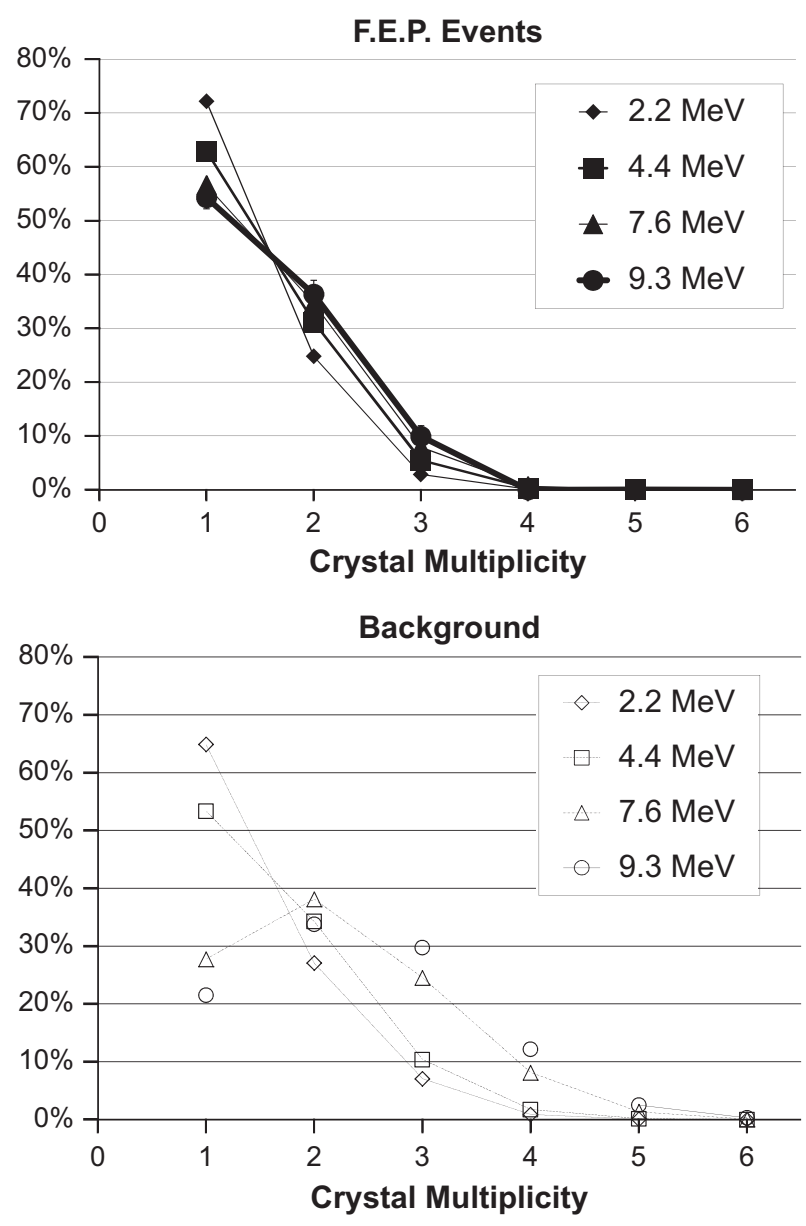

Fig. 6. Crystals multiplicity for FEP and background events. If not displayed error bars are smaller than symbol size.

Table 1

Cluster multiplicity for FEP and background events. Two AGATA triple clusters were used in the measurement.

\begin{tabular}{lll}
\hline \multicolumn{2}{l}{ Full Energy Peak (FEP) Events } & \\
\hline Energy (MeV) & $M_{\text {clust }}=1(\%)$ & $M_{\text {clust }}=2(\%)$ \\
2.2 & 92 & 8 \\
4.4 & 88 & 12 \\
7.6 & 85 & 15 \\
9.3 & 86 & 14 \\
Background & & $M_{\text {clust }}=2(\%)$ \\
\hline Energy (MeV) & $M_{\text {clust }}=1(\%)$ & 14 \\
\hline 2.2 & 86 & 20 \\
4.4 & 80 & 35 \\
7.6 & 65 & 42 \\
\hline .3 & 58 & \\
\hline
\end{tabular}



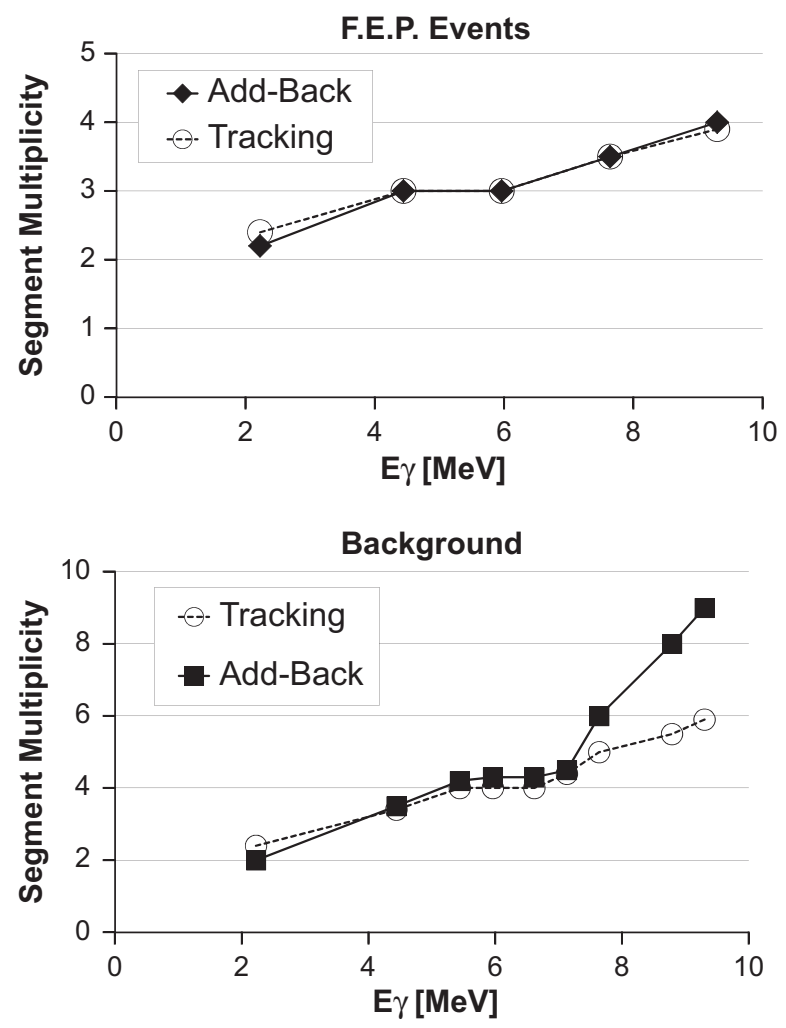

Fig. 7. Segments multiplicity as a function of gamma energy for FEP and background events. Results for gamma-ray tracking and standard "add-back" are compared. The centroids of each distribution are plotted with empty circles and black squares respectively. Error bars are smaller than symbol size.

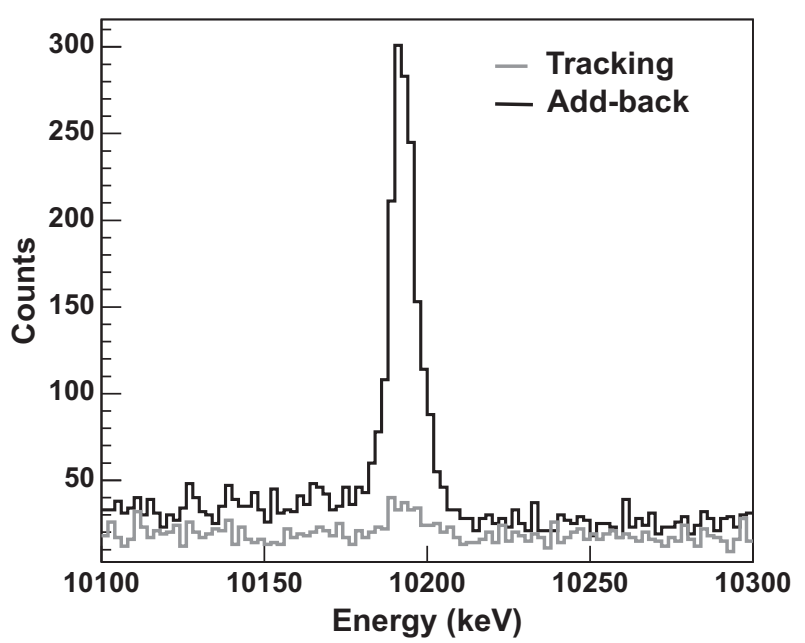

Fig. 8. Spectra obtained using the gamma-ray tracking algorithm (gray line) and the standard add-back one(black line) in the region around $10.196 \mathrm{MeV}$ (i.e. ${ }^{74} \mathrm{Ge}$ neutron separation energy).The peak that appears in the add-back spectrum is associated to the sum energy of the gamma rays emitted following the ${ }^{74} \mathrm{Ge}$ nucleus de-excitation, after neutron capture by ${ }^{73} \mathrm{Ge}$. These events are correctly recognized as composed by multiple gamma rays and disentangled by the tracking algorithm.

indeed suppressed in the "tracking" spectrum (gray line), while is clearly visible in the add-back one (black line).

This peak is associated to the sum energy of the gamma-rays emitted following the ${ }^{74} \mathrm{Ge}$ nucleus de-excitation, after neutron capture by ${ }^{73} \mathrm{Ge}$. The ground state decay from $10.196 \mathrm{MeV}$ level is not allowed [70,71], therefore the events in the peak have gamma multiplicities larger than one. As the tracking algorithm [69]

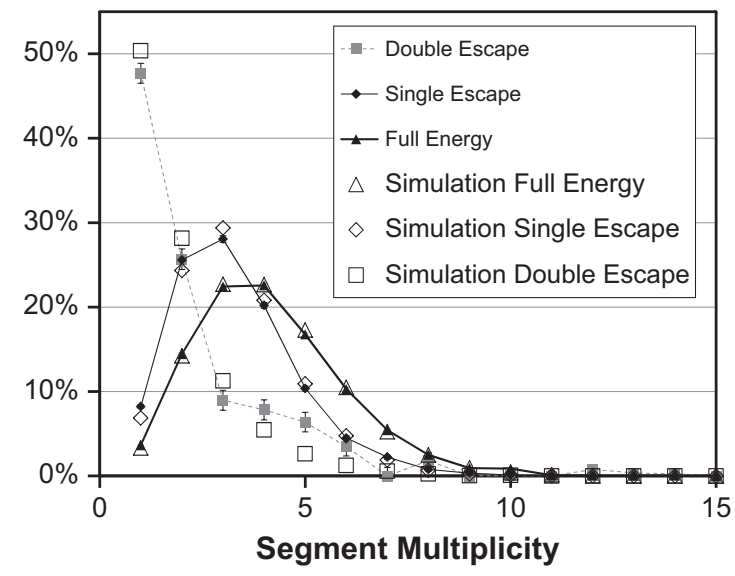

Fig. 9. Segments multipliity distributions for $7.6 \mathrm{MeV}$ gamma-rays (filled symbols). The case of FEP, SE, DE are compared. The resutls of the corresponding Geant4 simulations (add-back) are shown with open circles. If not displayed error bars are smaller than symbol size.

recognizes the peak as a sum-peak of two or more gamma-rays it is suppressed in the 'Tracking' spectrum.

In Fig. 9 the segment multiplicity distributions for the cases of full energy peak $\left(E_{\text {gamma }}=7.6 \mathrm{MeV}\right)$, single escape (S.E.) and double escape (D.E.) events are compared. The fact that the distributions have centroids shifted toward higher multiplicities for the case of full energy and single escape is caused by the presence of the $511 \mathrm{keV}$ gamma rays from pair production. In addition, the fact that $\sim 50 \%$ of double escape events have multiplicity larger than one can be attributed to the presence of Bremsstrahlung radiation and Compton interactions of the gamma-ray prior to the pair production. In Fig. 9 the results of Geant4 simulations [56,57] are also reported (open symbols), showing a good matching with the experimental data.

\section{Doppler correction of $15.1 \mathrm{MeV}$ gamma-rays}

In contrast to the $\mathrm{Am}-\mathrm{Be}-\mathrm{Fe}$ radioactive source data, the 15.1 MeV gamma rays are emitted by a ${ }^{12} \mathrm{C}$ nucleus moving at $v / c \sim 5 \%$ (see Section 2). Therefore the energy of the gamma rays detected in the laboratory system is shifted according to the equation:

$E_{\gamma, \text { shifted }}=E_{\gamma 0} \frac{(1-\beta)^{1 / 2}}{(1-\beta \cos \theta)}$

where $E_{\gamma 0}$ is the energy of the gamma-ray in the rest frame of the nucleus, $\beta$ is the velocity of the nucleus in the laboratory system relative to the speed of light and $\theta$ is the angle between the direction of motion of the nucleus and the direction of emission of the gamma-ray. While the angular distribution of the ${ }^{12} \mathrm{C}$ recoils is not measured by our detection system, with the AGATA detectors it is possible to determine the emission direction of the detected photon with different levels of precision, namely: (i) using the central position of the crystal with the largest energy deposit, (ii) the central position of the segment with the largest energy deposit, (iii) the position of the most energetic interaction point provided by the PSA algorithm [19] (from now on we refer to this procedure as "PSA+1HitID"), (iv) the incoming direction provided by the gamma-ray tracking algorithm [69].

The PSA + 1HitID algorithm calculates, for each event, the sum energy in all the detectors and determines the direction of the detected gamma-ray starting from the assumption that the first interaction corresponds to the location of the most energetic interaction [75], extracted by PSA algorithm [19]. 
This solution was chosen since the efficiency of the standard tracking algorithm [69] was found to significantly decrease in the $10-20 \mathrm{MeV}$ energy range. In particular, after applying the tracking algorithm [69] on both simulated and experimental data the ratio between the events in the $15.1 \mathrm{MeV}$ full energy peak for the tracked spectrum and the standard add-back with PSA+1HitID is 0.25 only. This is related to the fact that the used tracking algorithm was not optimized to treat gamma rays in the 10$20 \mathrm{MeV}$ range, where the pair production becomes the dominant interaction mechanism. In addition, in the present in-beam test the $15.1 \mathrm{MeV}$ gamma-ray is produced by the direct decay into the ground state of ${ }^{12} \mathrm{C}$, therefore the multiplicity is always one. This fact justifies the use of a simpler approach as the PSA+1HitID.

It is important to stress that the "multiplicity $=1$ " condition is fulfilled in several AGATA physics cases where the measurement of high-energy gamma rays is required (e.g. in the measurement of the Pygmy Dipole Resonance [1]).

In the used reaction (see Section 2) ${ }^{12} \mathrm{C}$ is produced with a $\beta$ of $\sim 5 \%$, however the velocity of the ${ }^{12} \mathrm{C}$ ions was not measured. Therefore, in order to Doppler correct in the optimal way the detected gamma-ray energy we determined the value of $\beta$ which better optimizes the centroid and width of the $15.1 \mathrm{MeV}$ full energy peak. In such a way we extracted an averaged velocity vector of magnitude $0.046(\beta)$ and components $(0,0.85,0.51)$ in the AGATA frame of reference; the AGATA reference frame is a right handed reference frame where the $z$ axis coincides with the optical axis of PRISMA (magnetic spectrometer of LNL-INFN lab) and the $x$ axis points downward (see Refs. [15,36,56,57]).

The components of the velocity vector are compatible with the beam direction. It is interesting to note that the best value of the extracted velocity is consistent with the results of simulations of the ${ }^{12} \mathrm{C}$ ion velocity distribution performed with PACE4 [72-74] giving a mean $\beta$ of 0.048 . More specifically we found that the $95 \%$ confidence interval for the $\beta$ value is between 0.042 and 0.058 and between $0^{\circ}$ and $10^{\circ}$ for the deviation angle with respect to the beam direction in the AGATA frame of reference.

The spectra in the region of $15 \mathrm{MeV}$ are shown in the panels of Fig. 10. In particular, different Doppler corrections were applied, using as gamma-ray emission direction the different options listed at the beginning of this section. In the top panel of Fig. 10 the spectrum obtained without Doppler correction (dashed black line) is compared to (i) the spectrum obtained by applying the Doppler correction using the central position of the segment with the largest energy deposit (thin black line) and (ii) the spectrum obtained by using the full information provided by the PSA "PSA + 1HitID" (thin gray line). By looking at the spectra displayed in the bottom panel of Fig. 10 one can note the marked improvement in the FWHM of the $15.1 \mathrm{MeV}$ peak passing from the spectrum obtained by applying the Doppler correction using the central position of the HPGe crystal with the largest energy deposit (i.e. detectors operated in standard mode, thick black line, FWHM larger than $160 \mathrm{keV}$ ) to the "PSA+1HitID" (gray line, $119 \mathrm{keV}$ FWHM, see also Table 1).

It is important to stress that, in this particular case, PSA techniques do not improve in a significant way the energy resolution as compared with the spectrum where Doppler correction was made using segment centers. In fact the FWHM slightly improves from 122 to $119 \mathrm{keV}$ (see Table 2). This fact is due to the uncertainty in ${ }^{12} \mathrm{C}$ ion vector velocity. The missing reconstruction on event by event basis of the ${ }^{12} \mathrm{C}$ ion velocity vector represents in this case the main limiting factor in the Doppler broadening correction capability.

In order to verify the different contributions to the final width $(119 \mathrm{keV})$ of the $15.1 \mathrm{MeV}$ peak Geant 4 simulation were performed and compared to the experimental result, see Fig. 11. This simulation was performed using the AGATA code $[56,57]$, applying then the same algorithm used to process the
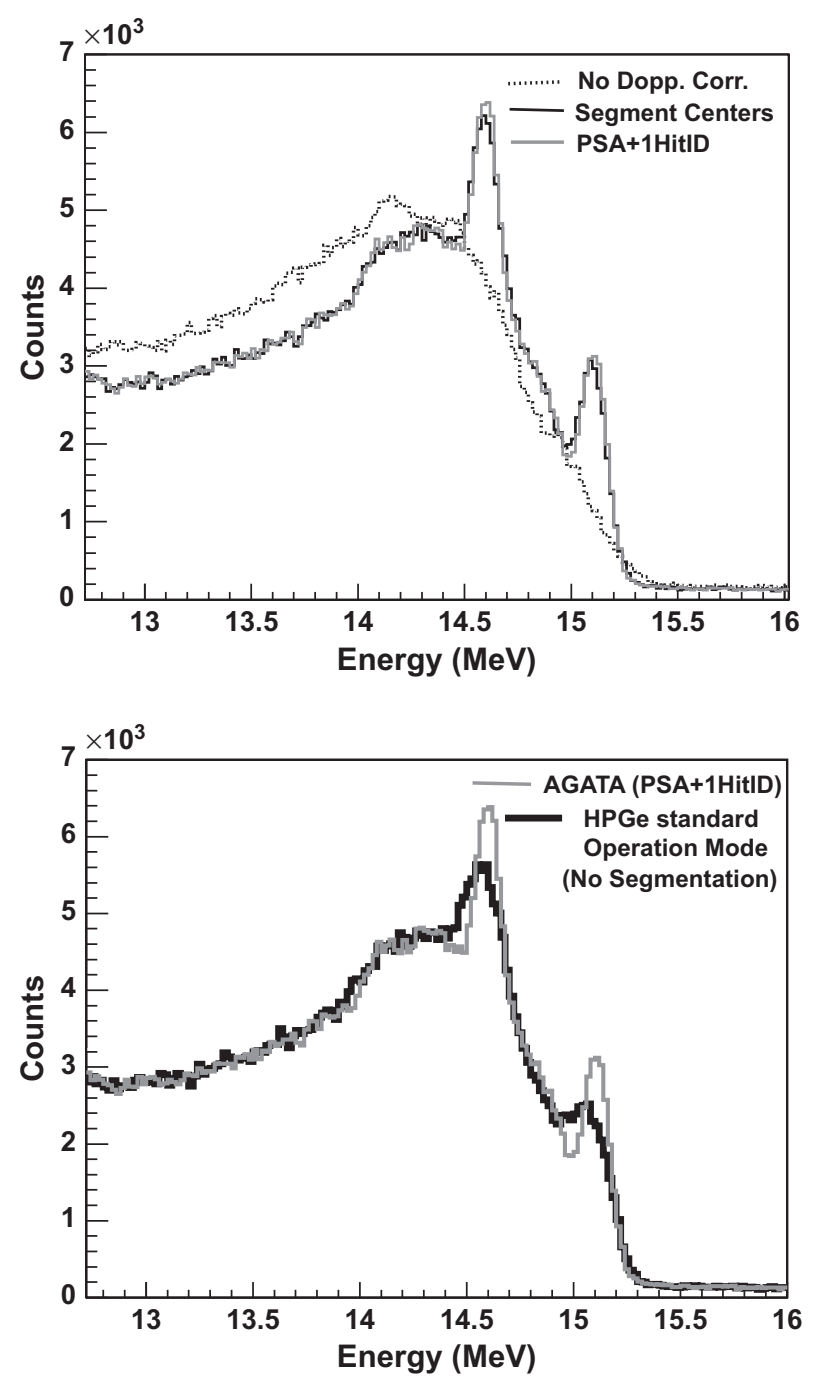

Fig. 10. Gamma-ray spectra acquired during the in-beam test, displayed in the region around $15 \mathrm{MeV}$. In the top panel the spectrum without Doppler correction (dashed black line) is compared to: (i) the spectrum obtained using only the central position of segments (thin black line) and (ii) the spectrum obtained using the PSA +1 HitID(thin gray line). In the bottom panel the spectra showing the performance of the detectors when operated in standard mode (Doppler correction using only the central position of the HPGe crystal with the largest energy deposit, thick black line) and using the PSA + 1HitID (gray line) are displayed.

\section{Table 2}

Values for the FWHM of the $15.1 \mathrm{MeV}$ gamma line obtained with Doppler correction using different position information, as described in the text. The main factor limiting the FWHM of the $15.1 \mathrm{MeV}$ gamma line was found to be the uncertainty due to the missing event by event reconstruction of the ${ }^{12} \mathrm{C}$ ion velocity vector. However, it is important to point out that considering the trend showed by the data displayed in Fig. 3, an intrinsic resolution of the order of $10 \mathrm{keV}$ should be expected at the energy of $15 \mathrm{MeV}$.

\begin{tabular}{lc}
\hline FWHM of $\mathbf{1 5 . 1} \mathbf{M e V}$ peak & \\
\hline PSA+1HitID & $119 \mathrm{keV}$ \\
Segments & $122 \mathrm{keV}$ \\
& $>160 \mathrm{keV}$ \\
Crystals & \\
\hline
\end{tabular}

experimental data. The ${ }^{12} \mathrm{C}$ ion velocity distribution was calculated using PACE4 [72-74] as discussed earlier. In the simulation the value of the intrinsic energy resolution of the detectors was extrapolated using the $E^{-1 / 2}$ law (see Fig. 3 ) and set to $8 \mathrm{keV}$ at 15.1 MeV. It should be pointed out, however, that this value has negligible impact on the final energy resolution obtained in the 


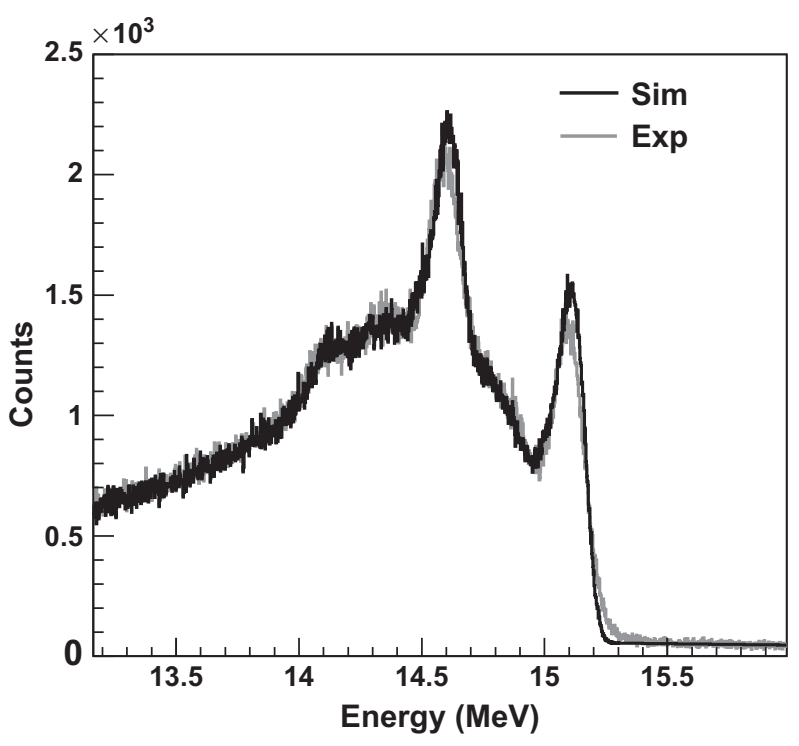

Fig. 11. Comparison between experimental (gray line) and simulated (black line) spectra in the $15 \mathrm{MeV}$ region. The main factor limiting the FWHM of the $15.1 \mathrm{MeV}$ gamma line was found to be the uncertainty due to the missing event by event reconstruction of the ${ }^{12} \mathrm{C}$ ion velocity vector.

experimental spectrum (see Table 2), since this is dominated by the Doppler broadening effect.

As can be noted in Fig. 11 there is good agreement between the measured and simulated curves, confirming that the measured FWHM of the Doppler corrected 15.1 MeV gamma line to $119 \mathrm{keV}$ is understood.

\section{Conclusions}

In this paper we studied the response of two AGATA triple clusters to gamma-rays in the energy range $2-15 \mathrm{MeV}$. The energy resolution was found to scale as $1 / \operatorname{sqrt}(E)$, once an accurate gain matching of the segments is performed. The linearity resulted to be better than $0.05 \%$ up to $10 \mathrm{MeV}$ and better than $0.1 \%$ up to $15.1 \mathrm{MeV}$. The experimental interaction multiplicity distributions show that, for high-energy gamma rays, background events are characterized on average by higher multiplicities than full energy peak ones. This is related to neutron capture events which characterize the spectrum for energy greater than $7 \mathrm{MeV}$. The multiplicity was compared with the results of Geant4 simulations. The Doppler corrected spectra were obtained for the $15.1 \mathrm{MeV}$ gamma line, using the PSA+1HitID procedure.

The main factor limiting the FWHM of the $15.1 \mathrm{MeV}$ gamma line was found to be the uncertainty due to the missing event by event reconstruction of the ${ }^{12} \mathrm{C}$ ion velocity vector. An intrinsic resolution of the order of $10 \mathrm{keV}$ should be expected at the energy of $15 \mathrm{MeV}$. The simple add-back and PSA+1HitID algorithm, in the case of the $15.1 \mathrm{MeV}$ gamma-rays, resulted to provide four times more counts in the full energy peak than the standard tracking algorithm. This is due to the fact that the $15.1 \mathrm{MeV}$ gamma-ray has multiplicity 1, the level of background is low and that the tracking algorithm was optimized in the energy range 0-4 MeV where Compton scattering dominates; at $15 \mathrm{MeV}$ the pair production is the main interaction mechanism instead. As in several AGATA physics cases which involve the measurement of high-energy gamma rays the "multiplicity $=1$ " condition is fulfilled, therefore the presented results might suggest a simple and efficient alternative to standard tracking, provided that the level of background radiation is sufficiently low.

\section{Acknowledgments}

This research has received funding from the European Union Seventh Framework Program FP7/2007-2013 under grant Agreement no. 262010-ENSAR. A.G. activity has been supported by the MINECO Spain, under grants AIC-D-2011-0746, FPA2011-29854 and by and Generalitat Valenciana, Spain, under grant PROMETEO/2010/101. We acknowledge the support by the German BMBF under Grants 06K-167 and 06KY205I.

\section{References}

[1] O. Wieland, et al., Physical Review Letters 102 (2009) 092502.

[2] R. Nicolini, et al., Acta Physica Polonica B 42 (3/4) (2011) 653.

[3] O. Wieland, et al., Physical Review Letters 97 (2006) 012501.

[4] A. Corsi, et al., Physics Letters B 679 (2009) 197.

[5] A. Corsi, et al., Physical Review C 84 (2011) 041304.

[6] G. Duchêne, et al., Nuclear Instruments \& Methods in Physics Research Section A 432 (1999) 90.

[7] J. Eberth, et al., Progress in Particle and Nuclear Physics 28 (1992) 495.

[8] J. Eberth, et al., Nuclear Instruments \& Methods in Physics Research Section A 369 (1996) 135.

[9] F. Beck, Progress in Particle and Nuclear Physics 28 (1992) 443.

[10] J. Simpson, Zeitschrift für Physik A 358 (1997) 139.

[11] M. Wilhelm, et al., Nuclear Instruments \& Methods in Physics Research Section A 381 (1996) 462.

[12] F. Camera, et al., Nuclear Instruments \& Methods in Physics Research Section A 351 (1994) 401.

[13] B. Million, et al., Nuclear Instruments \& Methods in Physics Research Section A 452 (2000) 422.

[14] S. Akkoyun, A. Algora, B. Alikhani, F. Ameil, G. de Angelis, L. Arnold, A. Astier, A. Atac, Y. Aubert, C. Aufranc, A. Austin, S. Aydin, F. Azaiez, S. Badoer, D. Balabanski, D. Barrientos, G. Baulieu, R. Baumann, D. Bazzacco, F. Beck, et al., Nuclear Instruments \& Methods in Physics Research Section A 668 (2012) 26.

[15] A. Gadea, et al., Nuclear Instruments \& Methods in Physics Research Section A 654 (2011) 88

[16] J. Eberth, J. Simpson, Progress in Particle and Nuclear Physics 60 (2008) 283.

[17] I.Y. Lee, M.A. Deleplanque, K. Vetter, Reports on Progress in Physics 66 (2003) 1095.

[18] I.Y. Lee, et al., Nuclear Physics A 746 (2004) 255C.

[19] R. Venturelli, D. Bazzacco, LNL Annual Report 2004, INFN-LNL, Legnaro, Italy, 2005, p. 220.

[20] A. Olariu, et al., IEEE Transactions on Nuclear Science 53 (2006) 1028.

[21] A. Olariu, Pulse Shape Analysis for the Gamma-ray Tracking Detector AGATA, Ph.D. Thesis, Université Paris-Sud 11, Orsay France, 2007.

[22] P. Désesquelles, et al., European Physical Journal A 40 (2009) 237.

[23] F.C.L. Crespi, HPGe Segmented Detectors in Gamma-ray Spectroscopy Experiments with Exotic Beams, Ph.D. Thesis, Universitá degli Studi di Milano, 2008 $\langle$ http://hdl.handle.net/2434/152528〉.

[24] F.C.L. Crespi, et al., Nuclear Instruments \& Methods in Physics Research Section A 570 (2007) 459.

[25] F.C.L. Crespi, et al., Nuclear Instruments \& Methods in Physics Research Section A 604 (2009) 604.

[26] M. Schlarb, Simulation and Real-Time Analysis of Pulse Shapes from Highly Segmented Germanium Detectors, Ph.D. Thesis, Technical University Munich, Munich, Germany, 2008 〈http://www.e12.physik.tu-muenchen.de/ groups/agata/ $>$.

[27] M. Schlarb, et al., European Physical Journal A 47 (2011) 132.

[28] T. Kröll, D. Bazzacco, Nuclear Instruments \& Methods in Physics Research Section A 565 (2006) 691.

[29] J. van der Marel, B. Cederwall, Nuclear Instruments \& Methods in Physics Research Section A 437 (1999) 538.

[30] G.J. Schmid, et al., Nuclear Instruments \& Methods in Physics Research Section A 430 (1999) 69.

[31] A. Lopez-Martens, et al., Nuclear Instruments \& Methods in Physics Research Section A 533 (2004) 454.

[32] D. Bazzacco, Nuclear Physics A 746 (2004) 248. (Proceedings of the 2029 Sixth International Conference on Radioactive Nuclear Beams (RNB6)).

[33] F. Recchia, In-beam Test and Imaging Capabilities of the AGATA Prototype Detector, Ph.D. Thesis, Università degli Studi di Padova, Padova, Italy, 2008 $\langle$ http://npgroup.pd.infn.it/Tesi/PhD-thesisRecchia.pdf $\rangle$.

[34] F. Recchia, et al., Nuclear Instruments \& Methods in Physics Research Section A 604 (2009) 555.

[35] F. Recchia, et al., Nuclear Instruments \& Methods in Physics Research Section A 604 (2009) 60. 
[36] P.-A. Söderström, et al., Nuclear Instruments \& Methods in Physics Research Section A 638 (2011) 96

[37] B. Bruyneel, P. Reiter, G. Pascovici, Nuclear Instruments \& Methods in Physics Research Section A 569 (2006) 764.

[38] B. Bruyneel, P. Reiter, G. Pascovici, Nuclear Instruments \& Methods in Physics Research Section A 569 (2006) 774.

[39] B. Bruyneel, et al., Nuclear Instruments \& Methods in Physics Research Section A 599 (2009) 196.

[40] B. Bruyneel, et al., Nuclear Instruments \& Methods in Physics Research Section A 608 (2009) 99.

[41] A. Wiens, H. Hess, B. Birkenbach, B. Bruyneel, J. Eberth, D. Lersch, G. Pascovici, P. Reiter, H. Thomas, Nuclear Instruments \& Methods in Physics Research Section A 618 (2010) 223.

[42] B. Bruyneel, et al., Nuclear Instruments \& Methods in Physics Research Section A 641 (2011) 92.

[43] B. Bruyneel, Detector Simulation Software ADL, unpublished, 〈http://www. ikp.uni-koeln.de/research/agata/index.php?showdownload $>$.

[44] M.R. Dimmock, et al., Characterisation results from an AGATA prototype detector, IEEE Transactions on Nuclear Science 56 (3) (2009) 1593-1599 (art. no. 5076035).

[45] M.R. Dimmock, et al., Validation of pulse shape simulations for an AGATA prototype detector, IEEE Transactions on Nuclear Science 56 (4) (2009) 2415-2425 (art. no. 5204764).

[46] T. Ha, et al., Nuclear Instruments \& Methods in Physics Research Section A 697 (2013) 96.

[47] T. Ha, Characterisation des detecteurs d'AGATAetEtude del'Hyperdeformation Nucleaire dansla Region deMasse 120, Ph.D. Thesis, UniversiteParis-Sud 11, Orsay, France, 2009.

[48] P. Désesquelles, Nuclear Instruments \& Methods in Physics Research Section A 654 (2011) 324

[49] F.C.L. Crespi, et al., Nuclear Instruments \& Methods in Physics Research Section A 593 (2008) 440.

[50] C. Domingo-Pardo, et al., Nuclear Instruments \& Methods in Physics Research Section A 643 (2011) 79

[51] N. Goel, et al., Nuclear Instruments \& Methods in Physics Research Section A A652 (2011) 591.

[52] A. Pullia, et al., Cross-talk limits of highly segmented semiconductor detectors, IEEE Transactions on Nuclear Science 58 (3 PART 3) (June 2011) 1201-1205 (Article number 5756680).
[53] C.E. Lehner, Z. He, G.F. Knoll, IEEE Transactions on Nuclear Science 50 (4) (2003).

[54] H.J. Wollersheim, et al., Nuclear Instruments \& Methods in Physics Research Section A 537 (2005) 637.

[55] S. Agostinelli, et al., Nuclear Instruments \& Methods in Physics Research Section A 506 (2003) 250

[56] E. Farnea, et al., Nuclear Instruments \& Methods in Physics Research Section A 621 (2010) 331.

[57]〈http://agata.pd.infn.it/〉 (Simulations)

[58] D.E. Alburger, D.H. Wilkinson, Physical Review C 5 (1972) 384.

[59] D.F. Measday, et al., Nuclear Physics A 45 (1963) 98.

[60] R.H. Howell, et al., Physical Review C 21 (1980) 1158.

[61] D. Bergholer, et al., Nuclear Physics A 263 (1976) 109.

[62] R. Nicolini, et al., Nuclear Instruments \& Methods in Physics Research Section A 582 (2007) 554

[63] F.G.A. Quarati, et al., Nuclear Instruments \& Methods in Physics Research Section A 629 (2011) 157.

64] A. Georgiev, W. Gast, IEEE Transactions on Nuclear Science 40 (1993) 770

[65] L. Arnold, et al., IEEE Transactions on Nuclear Science 53 (2006) 723.

[66] D. Bazzacco, et al., In preparation.

[67] B. Bruyneel, et al., LNL Annual Report 2010, INFN-LNL, Legnaro, Italy, 2011, pp. 64-65.

[68] A. Ataç, et al., Nuclear Instruments \& Methods in Physics Research Section A 607 (2009) 554

[69] D. Bazzacco, "mgt" (Mars Gamma Tracking) Code Developed Within the TMR (Training and Mobility through Research) Program 'Gamma-ray Tracking Detectors' for the MARS Prototype detector (see e.g. Th. Kröll et al., European Physical Journal A 20 (2003) 205)

[70] D.C. Kocher, Nuclear Data Sheets 17 (1976) 519

[71] J.-H. Chao, Applied Radiation and Isotopes: Including Data, Instrumentation and Methods for Use in Agriculture, Industry and Medicine 44 (1993) 605.

[72] 〈http://lise.nscl.msu.edu/lise.html 〉.

[73] A. Gavron, Physical Review C 21 (1980) 230.

[74] O.B. Tarasov, D. Bazin, Nuclear Instruments \& Methods in Physics Research Section B 204 (2003) 174

[75] O. Wieland, et al., Nuclear Instruments \& Methods in Physics Research Section A 487 (2002) 441 